\title{
Factores que Influyen en el Error Humano de los Trabajadores en Líneas de Montaje Manual
}

\author{
Yolanda A. Báez ${ }^{(1)}$, Manuel A. Rodríguez ${ }^{(2)}$, Enrique J. De la Vega ${ }^{(3)}$ y Diego A. Tlapa ${ }^{(1)}$ \\ (1) Universidad Autónoma de Baja California, Facultad de Ingeniería, Arquitectura y Diseño, \\ Carretera Tijuana-Ensenada Km. 103, Ensenada, Baja California-México (e-mail: yolanda@uabc.edu.mx, \\ diegotlapa@uabc.edu.mx) \\ (2) Instituto Tecnológico de Ciudad Juárez, Blvd. Tecnológico No. 1340, Ciudad Juárez, Chihuahua-México \\ (e-mail: manuel_rodriguez_itcj@yahoo.com) \\ (3) Instituto Tecnológico de Hermosillo, Ave. Tecnológico y Periférico Poniente S/N, Hermosillo, Sonora- \\ México (e-mail: e_delavega_mx@yahoo.com)
}

Recibido Mar. 28, 2013; Aceptado May. 27, 2013; Versión final recibida Jul. 11, 2013

\begin{abstract}
Resumen
Se describe el error humano determinando veintiocho factores que influyen en el comportamiento humano y que en su conjunto conforman las condiciones de operación bajo las que las personas trabajan. El error humano se encuentra en el origen de multitud de accidentes y, por lo tanto, es un elemento clave que debe ser evitado. Esto puede ser logrado si se conoce y explica el error mediante el análisis de los diferentes factores. Se analizan en un grupo de trabajadores de líneas de montaje manual cada uno de los factores. Luego se realiza un análisis de correlación múltiple con el objetivo de buscar los factores más influyentes. Esto permite reducir a nueve el número de factores a estudiar mediante la construcción de modelos de confiabilidad humana para en plantas de montaje.
\end{abstract}

Palabras clave: error humano, líneas de ensamble, comportamiento humano, accidentes laborales

\section{Factors Influencing the Human Error of Workers in Manual Assembly Lines}

\begin{abstract}
Human error is described by determining twenty eight factors that influence human behavior and which together make up the operating conditions under which people work. Human error is the source of numerous accidents and, therefore, is a key element that must be reduced or eliminated. This can be achieved if human error is known and explained through the analysis of the different factors that influence it. Each of these factors is analyzed in a group of workers from manual assembly lines. After that results are analyzed through multiple correlation analysis to find the most influential factors. This allowed reducing to nine the factors to be considered to develop human reliability models in assembly plants.
\end{abstract}

Keywords: human error, assembly lines, human behavior, labor accidents 


\section{INTRODUCCIÓN}

La complejidad de los sistemas está relacionada con la cantidad de información que describe al sistema. Para sistemas complejos, además de la confiabilidad tecnológica de los componentes del sistema es necesario considerar los aspectos tecnológicos de la información y las interacciones humanas (Yamamura et al., 1989). En muchos sistemas que involucran la interacción entre humanos y máquinas, una de las principales contribuciones a las probabilidades de falla se debe a sucesos provocados por seres humanos, según informa la serie de estadísticas (Bedford y Cook, 2001); mismos que son conocidos como "factores humanos" y se definen como las capacidades físicas y psicológicas de la persona, el entrenamiento y la experiencia, así como las condiciones en que ésta debe operar para alcanzar el propósito al que está destinado (Amendola, 2002).

En un mundo de procesos de producción altamente mecanizados, las acciones que deben seguir los operadores se especifican dentro de tolerancias establecidas, cualquier desviación a partir de estas tolerancias es considerada un error humano, lo cual puede perturbar seriamente el proceso de producción, por tal motivo tales errores deben evitarse debido a la pérdida económica asociada a la que conllevan (Bubb, 2005). Asimismo, Rasmussen (1990) expresa su preocupación porque a pesar de la creciente complejidad, tamaño y cambio en los sistemas técnicos de hoy en día, se seguirá dependiendo de la intervención humana para su operación segura; ésto complica el mantenimiento de la seguridad en sistemas complejos y permite la identificación de un error humano como causa de accidentes cada vez más importantes. En este sentido, Collins y Leathley (1995) enfatizan que los ingenieros de seguridad y analistas de confiabilidad por lo general prestan más atención a los equipos y a los procesos técnicos que a los factores sociales y psicológicos como mecanismos que predisponen a un error personal, dejando de lado el hecho que la interacción del ser humano con los artefactos, y muy especialmente con los sistemas complejos no es fácil, ya que se ha estimado que entre el 60 y $70 \%$ de los accidentes e incidentes que tienen lugar en los sistemas complejos tiene como causa primaria al error humano.

La relevancia de los errores humanos en situaciones de accidentes exige enfoques transparentes y explícitos para la evaluación del desempeño humano, en los análisis de confiabilidad cuantitativos sistemáticos (Zio et al., 2009)). Sin embargo, el análisis del error humano, de la confiabilidad de sus actos, es un tema de gran complejidad, en el que conviene tener presente que el ser humano actúa siempre por y desde un gran número de variables personales, organizacionales, situacionales y/o ambientales, que a menudo imposibilitan determinación definitiva de las causas (Sebastián, 2002). Las actuales técnicas para el análisis de la confiabilidad Humana (HRA, por sus siglas en Inglés) difieren principalmente en los factores a considerar en el análisis, en el impacto y el nivel de dependencia de los mismos. Todas estas distinciones son subjetivas y puede dar lugar a una diferencia de varios órdenes de magnitud en los resultados del HRA. Este subjetivismo se puede reducir con la adecuada selección de factores que afectan la dependencia entre las acciones humanas (Čepin, 2008). Adicionalmente, estudios sobre la actuación humana en accidentes han demostrado que la influencia de las condiciones en las que se realiza la tarea es mayor que las características de la tarea en si. Esto ha llevado un cambio en el enfoque de los errores humanos: si el contexto es el principal factor que influye en el rendimiento del error humano, entonces el contexto y la probabilidad de error humano debe ser modelado (Marseguerra, et al., 2006).

El objetivo del presente trabajo es conocer los factores que pueden influir en el cometido de errores por parte de los trabajadores de las líneas de ensamble manual, ésto se llevó a cabo mediante revisión de literatura de las diferentes corrientes que explican el error humano; así como una revisión de aquellos factores que están relacionados, obteniendo así un concentrado de factores, mismo que posteriormente se utilizó para obtener las condiciones de operación bajo las que estas personas trabajan. Ésto permitió identificar, mediante análisis de correlaciones de múltiples factores, cuales son los que tienen una alta influencia en la aparición del error. Esta información será de suma importancia para poder construir un modelo de confiabilidad humana, para el nivel operativo, que ayude a identificar variables explicativas y el grado de influencia que tienen sobre el cometido del error. Lo anterior, apoya lo mencionado por Alvis (2011) quien menciona que los estudios deben representar aspectos fundamentales de la aplicación de técnicas instrumentales de análisis, pues permite la posibilidad de desarrollar métodos mucho más reproducibles, confiables y rápidos.

\section{MATERIALES Y MÉTODOS}

El metodología empleada en este trabajo consta de cuatro fases: i) identificar los factores que pueden influir para que el ser humano cometa errores; ii) agrupar los factores que tienen relación entre sí; iii) obtener las condiciones de operación del trabajador; y iv) reducir el número de factores que explican el error humano. 


\section{Identificar los factores que pueden influir para que el ser humano cometa errores}

Para la primera fase del trabajo se revisaron las diferentes aproximaciones existentes para el análisis del error humano, las que, según Cárdenas (2009), se pueden se pueden clasificar en cuatro corrientes: a) Explicaciones fuera de la persona (EFP), b) Explicaciones adentro de la persona (EAP), c) Explicaciones desde la interacción persona - máquina (EDI) y d) Explicaciones desde la relación persona - contexto

\section{a) Explicaciones fuera de la persona (EFP)}

En esta corriente la explicación del error humano se realiza desde el sistema del que forma parte sin entrar en una explicación causal desde la propia persona. Con explicaciones más descriptivas que causales se utilizan, fundamentalmente, probabilidades de error y juicios de expertos. Las técnicas agrupadas dentro de esta corriente tienen como hipótesis base el considerar las acciones de cada operador humano desde el mismo punto de vista que el buen o mal funcionamiento de un mecanismo. Las tareas de los operadores se descomponen en tareas elementales de las cuales se evalúa la confiabilidad de manera similar a la de cualquier dispositivo técnico, con algunos ajustes ligados a la mayor variabilidad e interdependencia de la actividad humana. Estas técnica presentan algunos deficiencias debido a que simplifican en exceso la actividad humana, no contemplan el contexto en que la actividad se sitúa, se centra en el comportamiento anómalo de la persona y depende en exceso del juicio de los analistas. En la Figura 1, se concentran la teorías contempladas dentro de esta corriente así como los factores, que cada teoría considera, que influyen en el cometido del error humano.

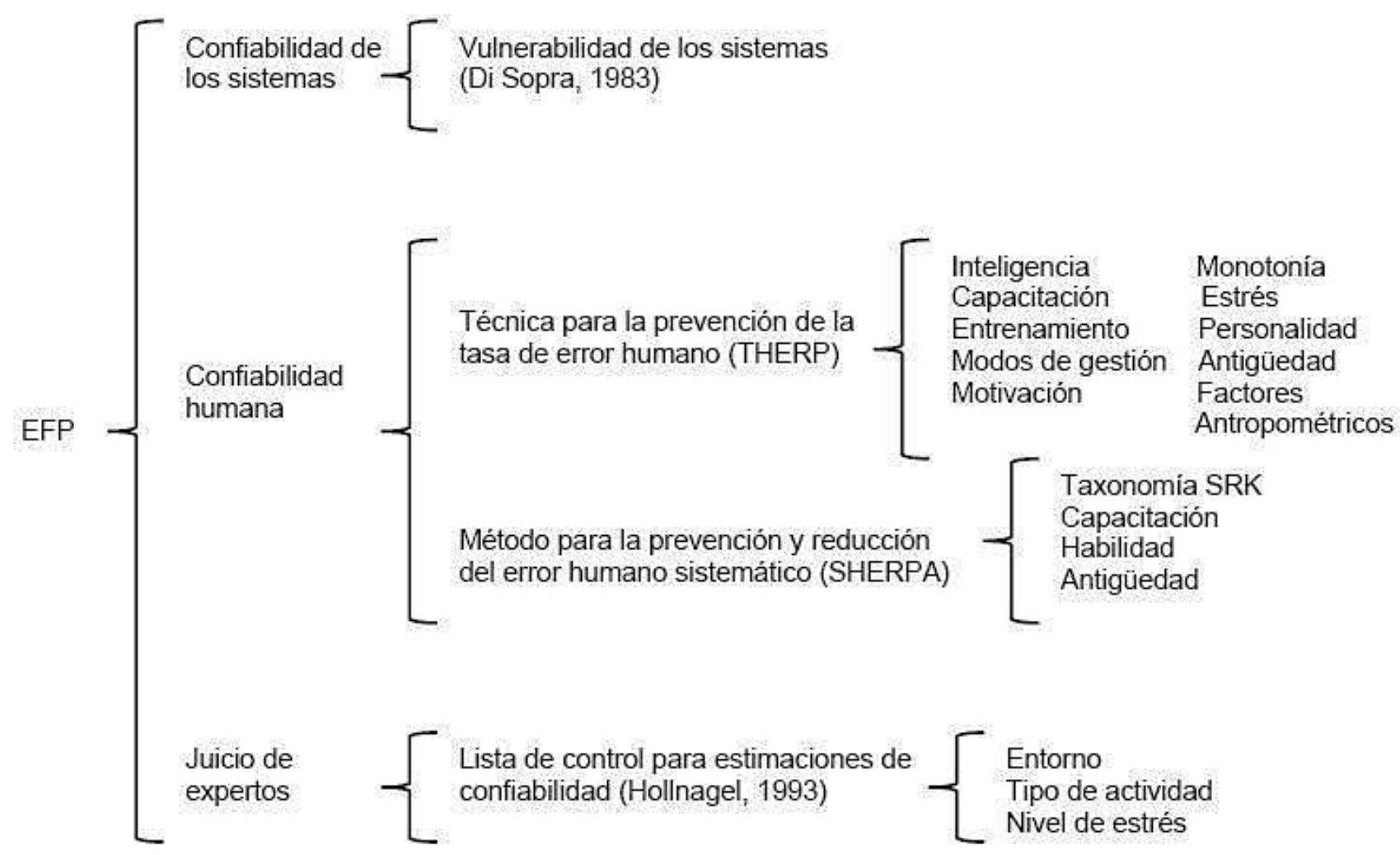

Fig. 1: Teorías y técnicas que explican el error desde fuera de la persona

b) Explicaciones adentro de la persona (EAP)

En la segunda corriente, la explicación del error humano se realiza desde la persona analizando algún atributo o componente causal. Las explicaciones toman un matiz causal derivado de los procesos cognitivos, la personalidad, la conducta y los patrones de acción. Las técnicas agrupadas bajo esta corriente (Figura 2), conciben el error desde los factores humanos; muestran un mayor potencial descriptivo que las técnicas anteriores, sin embargo conllevan la misma deficiencia de no contemplar el contexto donde se sitúa la actividad (Cárdenas, 2009). 


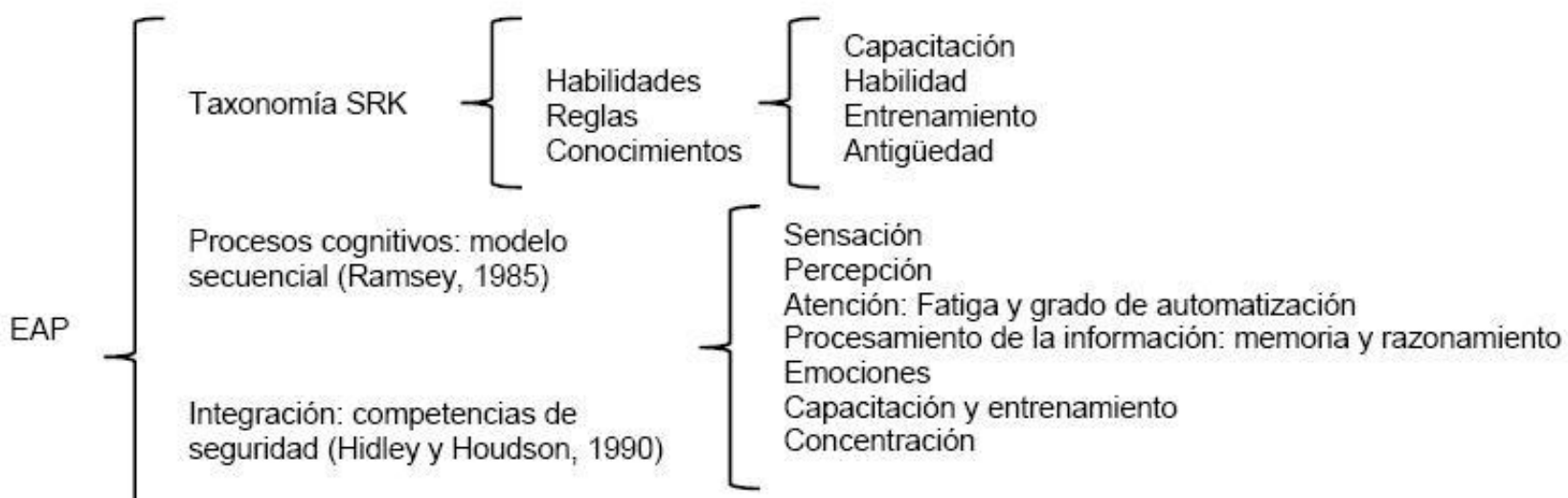

Personalidad y conducta $\{$ Personalidad

Fig. 2: Teorías y técnicas que explican el error desde adentro de la persona

c) Explicaciones desde la interacción persona - máquina (EDI)

Esta corriente toma en cuenta la interacción del operador humano con el artefacto; las explicaciones se basan sobre un componente o atributo del ser humano contemplado en su interacción con el sistema. En esta clasificación la ergonomía en general y la ergonomía cognitiva en particular han aportado importantes conceptos y teorías fundamentales para la comprensión del error humano. Los conceptos utilizados por las teorías y técnica de esta corriente, concentradas en la Figura 3, aportan una mejora al introducir específicamente la interacción del ser humano con otros elementos del sistema, sin embargo, para llegar a un análisis más completo del conjunto de variables que se relacionan con el error humano es necesario además contemplar las interacciones que ocurren que el proceso global de trabajo.

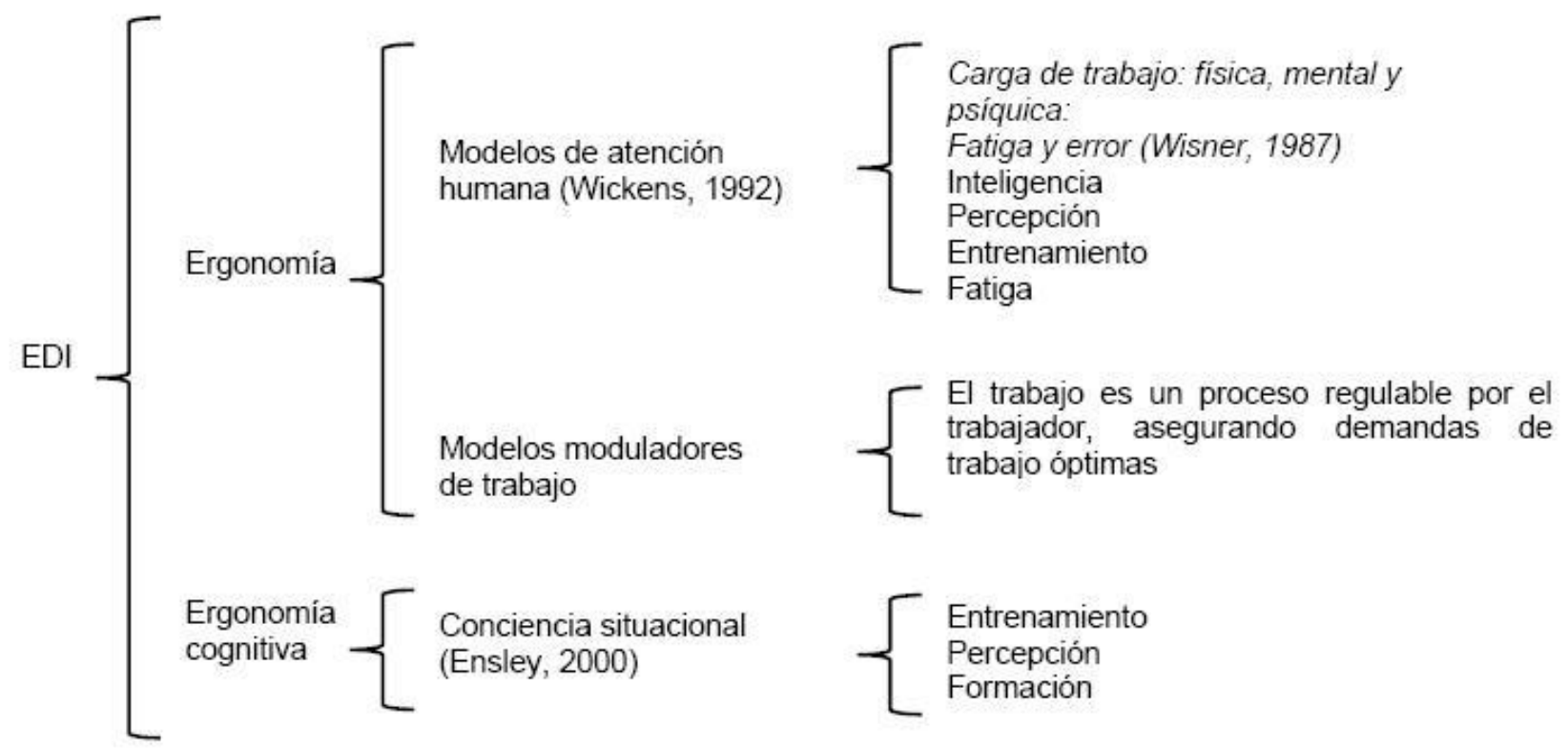

Fig. 3: Teorías y técnicas que explican el error desde la interacción de la persona

d) Explicaciones desde la relación persona - contexto

Las explicaciones parten de contexto, perdiendo su matriz de atributo para convertirse en relaciones. Dentro de esta corriente se consideran las nuevas tendencias referidas a la acción situada conocimiento socialmente distribuido. En esta corriente destaca la teoría de la actividad, que consiste en un conjunto de conocimientos, tecnologías y recursos encaminados al análisis, desarrollo y optimización de configuraciones de actividad humana (Sebastián, 2002), así como la patología residente, modelo que tiene la virtud de 
mostrar el modo en que los operadores se relacionan con el fallo de sistemas complejos e interactivos, produciendo un accidente. Desde esta perspectiva, los accidentes son consecuencias de las interacciones de una serie de fallas o defectos ya presentes en el sistema, muchas de las cuales no son visibles y tienen serias consecuencias posteriores. Una vez analizadas las teorías que se integran en cada una de las Corrientes que tratan de explicar el error humano, se procede a concentrar los factores que influyen para que éste aparezca, con el objetivo de identificar factores en común entre las teorías de cada corriente, obteniéndose un total de 28 factores diferentes tal como se muestra en la Tabla 1.

Tabla 1: Concentrado de factores de las diferentes corrientes que explican el error humano

\begin{tabular}{|c|c|c|c|c|c|c|c|}
\hline & $\begin{array}{l}\text { Error } \\
\text { Humano }\end{array}$ & $\begin{array}{c}\text { Taxonomía } \\
\text { srk }\end{array}$ & $\begin{array}{l}\text { Factores de } \\
\text { forma del } \\
\text { conocimiento }\end{array}$ & $\begin{array}{l}\text { Modelo } \\
\text { secuencial }\end{array}$ & $\begin{array}{l}\text { Competencia } \\
\text { de seguridad }\end{array}$ & Ergonomía & $\begin{array}{c}\text { Ergonomía } \\
\text { cognitiva }\end{array}$ \\
\hline \multicolumn{8}{|l|}{ Procesos mentales } \\
\hline \multicolumn{8}{|l|}{ Inteligencia } \\
\hline \multicolumn{8}{|l|}{ Percepción } \\
\hline \multicolumn{8}{|l|}{ Razonamiento } \\
\hline \multicolumn{8}{|l|}{ Atención } \\
\hline \multicolumn{8}{|l|}{ Emociones } \\
\hline \multicolumn{8}{|l|}{ Memoria } \\
\hline \multicolumn{8}{|l|}{ Concentración } \\
\hline \multicolumn{8}{|l|}{ Capacitación } \\
\hline \multicolumn{8}{|l|}{ Habilidad } \\
\hline \multicolumn{8}{|l|}{ Entrenamiento } \\
\hline \multicolumn{8}{|l|}{ Experiencia } \\
\hline \multicolumn{8}{|l|}{ Formación (escolaridad) } \\
\hline \multicolumn{8}{|l|}{ Factores organizacionales } \\
\hline \multicolumn{8}{|l|}{ Modos de gestión } \\
\hline \multicolumn{8}{|l|}{ Motivación } \\
\hline \multicolumn{8}{|l|}{ Comunicación } \\
\hline \multicolumn{8}{|l|}{$\begin{array}{l}\text { Carga de trabajo (física, } \\
\text { mental y psíquica) }\end{array}$} \\
\hline \multicolumn{8}{|l|}{ Monotonía } \\
\hline \multicolumn{8}{|l|}{ Factores antropométricos } \\
\hline \multicolumn{8}{|l|}{ Factores fisiológicos } \\
\hline \multicolumn{8}{|l|}{ Condiciones físicas } \\
\hline \multicolumn{8}{|l|}{ Velocidad de reacción } \\
\hline \multicolumn{8}{|l|}{ Coordinación psicomotora } \\
\hline \multicolumn{8}{|l|}{ Factores personales } \\
\hline \multicolumn{8}{|l|}{ Estrés } \\
\hline \multicolumn{8}{|l|}{ Personalidad } \\
\hline \multicolumn{8}{|l|}{ Responsabilidad } \\
\hline Antigüedad & & & & & & & \\
\hline Género & & & & & & & \\
\hline Edad & & & & & & & \\
\hline $\begin{array}{l}.-, \tilde{n}^{\star} \text { Estados } \\
\text { transitorios }\end{array}$ & & & & & & & \\
\hline Fatiga & & & & & & & \\
\hline ** Necesidad de desvío & & & & & & & \\
\hline
\end{tabular}

Agrupar los factores que se encuentran relacionados entre sí.

El siguiente paso consistió en agrupar los factores que, de acuerdo a la literatura, están relacionados. Se llama carga de trabajo a la influencia exterior al ser humano. Según sus características y capacidades individuales, ésta carga de trabajo se traduce en estrés para un respectivo ser humano. Mulder (1980) define la carga de trabajo en función del número de etapas de un proceso o en función del número de procesos para realizar correctamente la tarea y, más particularmente en función de los modos de gestión, factores antropométricos, niveles de fatiga y monotonía de la tarea. En la Figura 4 se concentran los factores que se encuentran relacionados con la carga de trabajo. 


Carga de trabajo $\left\{\begin{array}{l}\text { Estrés } \\ \text { Modos de gestión } \\ \text { Factores antropométricos } \\ \text { Nivel de fatiga } \\ \text { Monotonía de la tarea }\end{array}\right.$

Fig. 4: Factores relacionados con la carga de trabajo

La edad es el tiempo transcurrido a partir del nacimiento de un individuo. Durante su crecimiento el individuo sufre cambios previsibles; está bien aceptado, por la comunidad científica, que el proceso de envejecimiento conlleva una disminución a nivel general de todas las funciones del organismo reduciendo así la capacidad funcional (Timiras, 1997). Collins et al., concluyen, en un trabajo publicado en el 2004, que existe una disminución del nivel de condición física, la velocidad de reacción y las condiciones psicomotoras con la edad. En la Figura 5 se muestran los factores que guardan relación entre ellos.

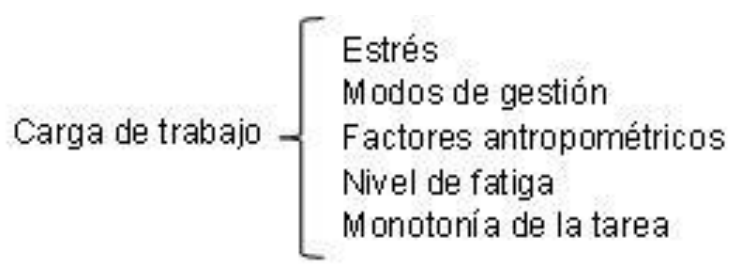

Fig. 5: Factores relacionados con la edad.

El concepto de experiencia generalmente se refiere al conocimientos procedimental (¿cómo hacer algo?), en lugar del conocimiento factual (¿Qué son las cosas?). Los filósofos tratan el conocimiento basado en la experiencia como "conocimiento empírico" o "un conocimiento a posteriori". El conocimiento procedimental esta íntimamente relacionado con el entrenamiento, el cual se refiere a la adquisición de conocimiento, habilidades y capacidades a través del tiempo y como resultado de la enseñanza de habilidades vocacionales y prácticas y conocimiento relacionado con actitudes que encierran cierta utilidad (capacitación). La Figura 6 muestra los factores que tienen relación con el factor experiencia.

Carga de trabajo $\left\{\begin{array}{l}\text { Estrés } \\ \text { Modos de gestión } \\ \text { Factores antropométricos } \\ \text { Nivel de fatiga } \\ \text { Monotonía de la tarea }\end{array}\right.$

Fig. 6: Factores relacionados con la experiencia

La personalidad puede sintetizarse como el conjunto de características o patrón de sentimientos, emociones y pensamientos ligados al comportamiento de cada individuo, que persiste a lo largo del tiempo frente a distintas situaciones y que lo hace diferente a los demás (Bermúdez, 2003). Actualmente, y resultado de una larga investigación y acumulación de conocimientos durante años, se acepta como modelo más cercano al constructo de personalidad al Modelo de los cinco grandes, en el cual se considera que los factores que subyacen a la personalidad son: la extraversión, neuroticismo, amabilidad, apertura a la experiencia y la responsabilidad (McCrae, 1999). La figura 7 agrupa los factores que guardan relación con el factor personalidad.

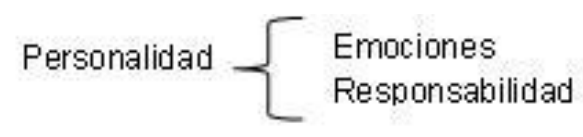

Fig. 7: Factores relacionados con la personalidad

Una vez realizada la agrupación de factores de acuerdo a la revisión de literatura, se logró obtener un a reducción significativa de 28 a 15 factores, sin dejar fuera ningún de los conceptos considerados por las corriente que explican el origen del error humano. En la tabla 2 se concentran los factores que tienen influencia en el cometido de errores por parte del humano. 
Tabla 2: Concentrado de factores que explican el error humano

\begin{tabular}{|l|l|}
\hline Atención & Edad \\
\hline Concentración & Experiencia \\
\hline Habilidad & Género \\
\hline Percepción & Formación \\
\hline Memoria & Motivación \\
\hline Razonamiento & Comunicación \\
\hline Inteligencia & Carga de trabajo \\
\hline Personalidad & \\
\hline
\end{tabular}

\section{Obtener las condiciones de operación del trabajador}

Tomando como referencia los factores obtenidos en el punto anterior, se procedió a evaluar cada uno de ellos en 30 operadores de líneas de ensamble manual, de una compañía electrónica del noroeste de México, para lo cual fue necesario conocer su información personal como la edad, género, experiencia, formación; así como aplicar una serie de pruebas psicométricas para conocer su información intrínseca como la personalidad, inteligencia, percepción, razonamiento, atención, memoria, concentración, habilidad, motivación, comunicación.

El estudio se realizó a un grupo de 18 personas del primer turno y 12 personas del segundo turno. Las escalas que se utilizaron para evaluar a cada uno de los factores se encuentran concentrados en la tabla 3.

Tabla 3: Escalas consideradas para cada uno de los factores

\begin{tabular}{|c|c|c|c|c|c|c|c|c|c|c|}
\hline \multicolumn{3}{|c|}{ Inteligencia (in) } & \multicolumn{3}{|c|}{ Memoria (me) } & \multicolumn{3}{|c|}{ Atención (at) } & \multicolumn{2}{|c|}{ Edad (ed) } \\
\hline $\mathrm{S}$ & Superior & 1 & $\mathrm{~S}$ & Superior & 1 & $S$ & Superior & 1 & 18 - 22 años & 1 \\
\hline STM & $\begin{array}{l}\text { Superior al } \\
\text { término medio }\end{array}$ & 2 & STM & $\begin{array}{l}\text { Superior al } \\
\text { término medio }\end{array}$ & 2 & STM & $\begin{array}{l}\text { Superior al } \\
\text { término medio }\end{array}$ & 2 & 22 - 26 años & 2 \\
\hline TM & Término medio & 3 & TM & Término medio & 3 & TM & $\begin{array}{l}\text { Término } \\
\text { medio }\end{array}$ & 3 & 26 - 32 años & 3 \\
\hline ITM & $\begin{array}{l}\text { Inferior al } \\
\text { término medio }\end{array}$ & 4 & ITM & $\begin{array}{l}\text { Inferior al } \\
\text { término medio }\end{array}$ & 4 & ITM & $\begin{array}{l}\text { Inferior al } \\
\text { término medio }\end{array}$ & 4 & 32 - 36 años & 4 \\
\hline I & Inferior & 5 & I & Inferior & 5 & I & Inferior & 5 & $\begin{array}{l}\text { Mas de } 36 \\
\text { años }\end{array}$ & 5 \\
\hline \multicolumn{3}{|c|}{ Motivación (mo) } & \multicolumn{3}{|c|}{ Concentración (cn) } & \multicolumn{3}{|c|}{ Habilidad (ha) } & \multicolumn{2}{|c|}{ Experiencia (ex) } \\
\hline A & Alta & 1 & $S$ & Superior & 1 & $\mathrm{~S}$ & Superior & 1 & $0-3$ meses & 1 \\
\hline SM & $\begin{array}{l}\text { Superior a la } \\
\text { media }\end{array}$ & 2 & STM & $\begin{array}{l}\text { Superior al } \\
\text { término medio }\end{array}$ & 2 & STM & $\begin{array}{l}\text { Superior al } \\
\text { término medio }\end{array}$ & 2 & $3-6$ meses & 2 \\
\hline $\mathrm{M}$ & Media & 3 & TM & Término medio & 3 & TM & $\begin{array}{l}\text { Término } \\
\text { medio }\end{array}$ & 3 & $\begin{array}{l}6 \text { meses - } 1 \\
\text { año }\end{array}$ & 3 \\
\hline IM & $\begin{array}{l}\text { Inferior a la } \\
\text { media }\end{array}$ & 4 & ITM & $\begin{array}{l}\text { Inferior al } \\
\text { término medio }\end{array}$ & 4 & ITM & $\begin{array}{l}\text { Inferior al } \\
\text { término medio }\end{array}$ & 4 & $1-3$ años & 4 \\
\hline B & Baja & 5 & I & Inferior & 5 & I & Inferior & 5 & $\begin{array}{l}\text { Mas de } 3 \\
\text { años }\end{array}$ & 5 \\
\hline \multicolumn{3}{|c|}{ Percepción (pc) } & \multicolumn{3}{|c|}{ Razonamiento (ra) } & \multicolumn{3}{|c|}{ Formación (fo) } & \multicolumn{2}{|c|}{ Género (ge) } \\
\hline$S$ & Superior & 1 & $S$ & Superior & 1 & \multicolumn{2}{|c|}{ Primaria } & 1 & Mujer & 1 \\
\hline STM & $\begin{array}{l}\text { Superior al } \\
\text { término medio }\end{array}$ & 2 & STM & $\begin{array}{l}\text { Superior al } \\
\text { término medio }\end{array}$ & 2 & \multicolumn{2}{|c|}{ Secundaria } & 2 & Hombre & 2 \\
\hline TM & Término medio & 3 & TM & Término medio & 3 & \multicolumn{2}{|c|}{ Preparatoria } & 3 & \multicolumn{2}{|c|}{$\begin{array}{l}\text { Comunicación } \\
(\mathrm{cm})\end{array}$} \\
\hline ITM & $\begin{array}{l}\text { Inferior al } \\
\text { término medio }\end{array}$ & 4 & ITM & $\begin{array}{l}\text { Inferior al } \\
\text { término medio }\end{array}$ & 4 & \multicolumn{2}{|c|}{ Carrera técnica } & 4 & Escasa & 1 \\
\hline I & Inferior & 5 & I & Inferior & 5 & \multicolumn{2}{|c|}{ Licenciatura } & 5 & Suficiente & 2 \\
\hline \multicolumn{3}{|c|}{ Personalidad (ps) } & & & & & & & & \\
\hline \multicolumn{2}{|l|}{ Tipo A } & 1 & & & & & & & & \\
\hline \multicolumn{2}{|c|}{ Tipo A - B } & 2 & & & & & & & & \\
\hline \multicolumn{2}{|l|}{ Tipo B } & 3 & & & & & & & & \\
\hline
\end{tabular}


Como primera prueba se evaluó en los trabajadores los factores atención, concentración, habilidad, percepción, memoria y razonamiento, obteniéndose los resultados concentrados en la tabla 4.

Tabla 4: Evaluación de los primeros seis factores a los trabajadores de línea de ensamble manual

\begin{tabular}{|c|c|c|c|c|c|c|c|}
\hline \multirow{2}{*}{ Trabajador } & \multicolumn{6}{|c|}{ Factores } & Turno \\
\hline & Atención & Concentración & Habilidad & Percepción & Memoria & Razonamiento & \multirow{19}{*}{ 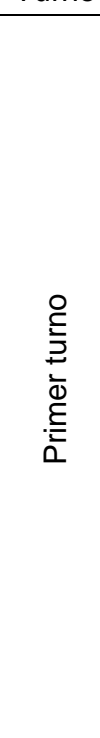 } \\
\hline A & 2 & 1 & 1 & 2 & 1 & 2 & \\
\hline$B$ & 1 & 1 & 2 & 1 & 2 & 2 & \\
\hline C & 2 & 2 & 3 & 1 & 1 & 1 & \\
\hline $\mathrm{D}$ & 2 & 2 & 2 & 1 & 1 & 2 & \\
\hline$E$ & 2 & 1 & 2 & 1 & 2 & 1 & \\
\hline $\mathrm{F}$ & 1 & 2 & 1 & 2 & 2 & 2 & \\
\hline $\mathrm{G}$ & 2 & 1 & 2 & 3 & 3 & 3 & \\
\hline $\mathrm{H}$ & 3 & 3 & 3 & 1 & 3 & 2 & \\
\hline $\mathrm{I}$ & 3 & 3 & 3 & 1 & 2 & 1 & \\
\hline $\mathrm{J}$ & 1 & 1 & 1 & 1 & 2 & 1 & \\
\hline $\mathrm{K}$ & 1 & 1 & 1 & 2 & 2 & 2 & \\
\hline $\mathrm{L}$ & 4 & 3 & 3 & 1 & 1 & 1 & \\
\hline $\bar{M}$ & 2 & 1 & 2 & 3 & 3 & 3 & \\
\hline $\mathrm{N}$ & 1 & 2 & 1 & 1 & 2 & 1 & \\
\hline $\mathrm{O}$ & 2 & 2 & 2 & 5 & 3 & 3 & \\
\hline $\mathrm{P}$ & 4 & 5 & 3 & 1 & 1 & 1 & \\
\hline$Q$ & 2 & 2 & 3 & 2 & 2 & 1 & \\
\hline$R$ & 3 & 3 & 3 & 1 & 2 & 2 & \\
\hline$S$ & 1 & 1 & 2 & 2 & 3 & 3 & \multirow{12}{*}{ 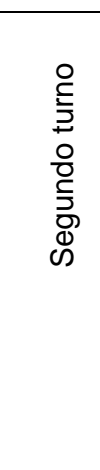 } \\
\hline$T$ & 2 & 1 & 1 & 3 & 2 & 2 & \\
\hline$U$ & 3 & 3 & 3 & 1 & 2 & 1 & \\
\hline $\mathrm{V}$ & 4 & 5 & 3 & 3 & 2 & 2 & \\
\hline $\mathrm{W}$ & 4 & 3 & 3 & 3 & 2 & 3 & \\
\hline $\mathrm{X}$ & 3 & 3 & 3 & 1 & 2 & 1 & \\
\hline$Y$ & 1 & 2 & 1 & 1 & 1 & 1 & \\
\hline Z & 1 & 2 & 2 & 2 & 2 & 2 & \\
\hline AA & 3 & 3 & 3 & 4 & 4 & 4 & \\
\hline BB & 2 & 2 & 2 & 2 & 2 & 2 & \\
\hline CC & 2 & 1 & 2 & 1 & 2 & 2 & \\
\hline $\mathrm{DD}$ & 1 & 2 & 2 & 1 & 1 & 1 & \\
\hline
\end{tabular}

Para obtener las condiciones de operación del trabajador de línea se evaluó en cada trabajador cada uno de los factores, obteniéndose los resultados contenidos en la Tabla 5.

Tabla 5: Concentrado de las condiciones de operación de los trabajadores

\begin{tabular}{|c|c|c|c|c|c|c|c|c|c|c|c|}
\hline \multirow{2}{*}{ Trabajador } & \multicolumn{10}{|c|}{ Factores } & \\
\hline & in & at & ps & ed & ex & ge & fo & $\mathrm{mo}$ & $\mathrm{co}$ & $\mathrm{me}$ & \multirow{19}{*}{ 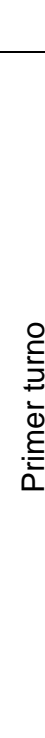 } \\
\hline A & 3 & 2 & 3 & 2 & 3 & 1 & 3 & 2 & 1 & 1 & \\
\hline$B$ & 3 & 1 & 3 & 3 & 3 & 1 & 2 & 3 & 1 & 2 & \\
\hline $\mathrm{C}$ & 3 & 2 & 2 & 3 & 4 & 1 & 2 & 3 & 1 & 1 & \\
\hline D & 3 & 2 & 2 & 4 & 1 & 1 & 2 & 2 & 2 & 1 & \\
\hline$E$ & 3 & 2 & 3 & 4 & 3 & 1 & 2 & 3 & 1 & 2 & \\
\hline$F$ & 3 & 1 & 3 & 2 & 1 & 1 & 3 & 2 & 1 & 2 & \\
\hline $\mathrm{G}$ & 3 & 2 & 3 & 2 & 2 & 2 & 3 & 2 & 2 & 3 & \\
\hline $\mathrm{H}$ & 3 & 3 & 3 & 3 & 4 & 2 & 2 & 3 & 2 & 3 & \\
\hline I & 3 & 3 & 3 & 2 & 3 & 2 & 3 & 4 & 1 & 2 & \\
\hline $\mathrm{J}$ & 3 & 1 & 3 & 4 & 3 & 2 & 3 & 2 & 1 & 2 & \\
\hline $\mathrm{K}$ & 3 & 1 & 1 & 2 & 3 & 2 & 2 & 2 & 2 & 2 & \\
\hline $\mathrm{L}$ & 3 & 4 & 2 & 3 & 2 & 2 & 2 & 3 & 2 & 1 & \\
\hline $\mathrm{M}$ & 3 & 2 & 3 & 3 & 1 & 1 & 2 & 4 & 1 & 3 & \\
\hline $\mathrm{N}$ & 3 & 1 & 2 & 2 & 4 & 1 & 3 & 3 & 2 & 2 & \\
\hline $\mathrm{O}$ & 3 & 2 & 2 & 3 & 3 & 2 & 3 & 3 & 2 & 3 & \\
\hline$P$ & 3 & 4 & 2 & 2 & 4 & 2 & 2 & 3 & 1 & 1 & \\
\hline $\mathrm{Q}$ & 3 & 2 & 3 & 2 & 1 & 1 & 2 & 4 & 1 & 2 & \\
\hline $\mathrm{R}$ & 3 & 3 & 3 & 2 & 4 & 2 & 3 & 2 & 1 & 2 & \\
\hline
\end{tabular}


Tabla 5: Continuación

\begin{tabular}{|c|c|c|c|c|c|c|c|c|c|c|c|}
\hline$S$ & 3 & 1 & 2 & 3 & 4 & 1 & 3 & 2 & 2 & 3 & \multirow{12}{*}{ 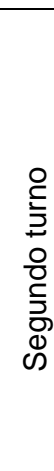 } \\
\hline $\mathrm{T}$ & 3 & 2 & 3 & 4 & 2 & 1 & 3 & 3 & 1 & 2 & \\
\hline $\mathrm{U}$ & 3 & 3 & 3 & 4 & 5 & 2 & 2 & 3 & 2 & 2 & \\
\hline $\mathrm{V}$ & 3 & 4 & 2 & 2 & 2 & 2 & 2 & 3 & 1 & 2 & \\
\hline W & 3 & 4 & 2 & 3 & 3 & 1 & 3 & 4 & 1 & 2 & \\
\hline $\bar{X}$ & 3 & 3 & 3 & 3 & 5 & 2 & 2 & 4 & 2 & 2 & \\
\hline$Y$ & 3 & 1 & 3 & 2 & 5 & 1 & 2 & 3 & 1 & 1 & \\
\hline Z & 3 & 1 & 3 & 2 & 3 & 1 & 3 & 4 & 2 & 2 & \\
\hline AA & 3 & 3 & 1 & 2 & 1 & 2 & 3 & 2 & 2 & 4 & \\
\hline BB & 3 & 2 & 1 & 4 & 4 & 1 & 2 & 2 & 2 & 2 & \\
\hline $\mathrm{CC}$ & 3 & 2 & 3 & 2 & 5 & 1 & 2 & 3 & 1 & 2 & \\
\hline DD & 3 & 1 & 2 & 3 & 3 & 1 & 3 & 4 & 2 & 1 & \\
\hline
\end{tabular}

Reducir el número de factores que explican el error humano

Para reducir el número de variables a considerar en un modelo que explican el error humano se aplicó un análisis de correlación de múltiples variables de los datos contenidos en la tabla 3; como resultado se observó que existe asociación entre los factores atención, concentración y habilidad, así como entre los factores percepción, memoria y razonamiento. Por lo que no resulta necesario integrarlos todos al modelo, los resultados se muestra en la tabla 6.

Tabla 6: Análisis de correlación de múltiples variables para seis factores

\begin{tabular}{|l|r|r|r|r|r|r|}
\hline & \multicolumn{1}{|c|}{ Atención } & \multicolumn{1}{c|}{ Concentración } & \multicolumn{1}{c|}{ Habilidad } & Percepción & \multicolumn{1}{c|}{ Memoria } & Razonamiento \\
\hline Atención & 1.0000 & 0.7745 & 0.7760 & 0.0954 & 0.0000 & 0.0338 \\
\hline Concentración & 0.7745 & 1.0000 & 0.6829 & -0.0352 & -0.1259 & -0.1620 \\
\hline Habilidad & 0.7760 & 0.6829 & 1.0000 & -0.0410 & 0.1173 & -0.0087 \\
\hline Percepción & 0.0954 & -0.0352 & -0.0410 & 1.0000 & 0.6111 & 0.7776 \\
\hline Memoria & 0.0000 & -0.1259 & 0.1173 & 0.6111 & 1.0000 & 0.7237 \\
\hline Razonamiento & 0.0338 & -0.1620 & -0.0087 & 0.7776 & 0.7237 & 1.0000 \\
\hline
\end{tabular}

Posteriormente se realiza de nuevo un análisis de correlaciones múltiples esperando encontrar nuevas relaciones entre factores, encontrándose que los factores atención e inteligencia tienen una buena asociación, así como personalidad y comunicación, tal como se muestra en las Tablas 7 y 8.

Tabla 7: Análisis de correlación de múltiples variables para diez factores

\begin{tabular}{|l|r|r|r|r|r|}
\hline & Inteligencia & \multicolumn{1}{|c|}{ Atención } & Personalidad & \multicolumn{1}{c|}{ Edad } & Experiencia \\
\hline Inteligencia & 1.000 & -0.364 & 0.182 & -0.219 & -0.170 \\
\hline Atención & -0.364 & 1.000 & -0.116 & -0.029 & -0.004 \\
\hline Personalidad & 0.182 & -0.116 & 1.000 & -0.017 & 0.101 \\
\hline Edad & -0.219 & -0.029 & -0.117 & 1.000 & 0.044 \\
\hline Experiencia & -0.170 & -0.004 & 0.101 & 0.044 & 1.000 \\
\hline Género & -0.198 & 0.525 & -0.107 & -0.134 & 0.084 \\
\hline Formación & 0.076 & -0.222 & 0.047 & -0.196 & -0.185 \\
\hline Motivación & 0.275 & 0.200 & 0.293 & -0.046 & 0.075 \\
\hline Comunicación & 0.216 & -0.089 & -0.452 & 0.150 & 0.082 \\
\hline Memoria & 0.153 & 0.000 & -0.068 & -0.059 & -0.219 \\
\hline
\end{tabular}

Tabla 8: Análisis de correlación de múltiples variables para diez factores

\begin{tabular}{|l|r|r|r|r|r|}
\hline & \multicolumn{1}{|c|}{ Género } & \multicolumn{1}{c|}{ Formación } & \multicolumn{1}{c|}{ Motivación } & \multicolumn{1}{c|}{ Comunicación } & Memoria \\
\hline Inteligencia & -0.198 & 0.076 & 0.275 & 0.216 & 0.153 \\
\hline Atención & 0.525 & -0.222 & 0.200 & -0.089 & 0.000 \\
\hline Personalidad & -0.107 & 0.047 & 0.293 & -0.452 & -0.068 \\
\hline Edad & -0.134 & -0.196 & -0.046 & 0.150 & -0.059 \\
\hline Experiencia & 0.084 & -0.185 & 0.075 & 0.082 & -0.219 \\
\hline Género & 1.000 & -0.009 & -0.153 & 0.261 & 0.276 \\
\hline Formación & -0.009 & 1.000 & -0.143 & 0.062 & 0.274 \\
\hline Motivación & -0.153 & -0.143 & 1.000 & -0.143 & -0.122 \\
\hline Comunicación & 0.261 & 0.062 & -0.143 & 1.000 & 0.274 \\
\hline Memoria & 0.276 & 0.274 & -0.122 & 0.274 & 1.000 \\
\hline
\end{tabular}




\section{ANÁLISIS DE RESULTADOS}

Mediante el primer análisis de correlación de efectuado entre seis factores se puede ver que existe fuerte relación entre las variables atención, concentración y habilidad; así como una fuerte correlación entre los factores percepción, memoria y razonamiento, por lo que se reduce a 11 la cantidad de factores (Tabla 9) que pueden explicar el error humano.

Tabla 9: Concentrado de factores de 11 factores que explican pueden explicar el error humano

\begin{tabular}{|l|l|}
\hline Inteligencia & Formación \\
\hline Atención & Motivación \\
\hline Personalidad & Comunicación \\
\hline Edad & Memoria \\
\hline Experiencia & Carga de trabajo \\
\hline Género & \\
\hline
\end{tabular}

Derivado del último análisis realizado al concentrado de resultados de la tabla 4 y en base a los resultados concentrados en las tablas 6 y 7, se pudo observar que los factores atención e inteligencia se encuentra relacionados, así como los factores comunicación y personalidad, motivo por el cual se decide dejar al factor inteligencia entre los 9 factores finales que explican el error humano (Tabla 10). Aún cuando los factores género y atención presentan una fuerte relación, se opta por dejar el factor género en el concentrado de factores puesto que los factores género e inteligencia no presentan correlación significativa y el factor inteligencia explicará al factor atención dentro del modelo.

Tabla 10: Concentrado de factores que integrarán el modelo de Confiabilidad humana

\begin{tabular}{|l|l|}
\hline Inteligencia & Formación \\
\hline Personalidad & Motivación \\
\hline Edad & Memoria \\
\hline Experiencia & Carga de trabajo \\
\hline Género & \\
\hline
\end{tabular}

\section{CONCLUSIONES}

El estudio realizado es de suma importancia ya que mediante la serie de prueba presentadas se logró reducir de 28 factores identificados de las cuatro corrientes filosóficas que tratan de explicar el error humano, a 9 factores sin sacrificar información importante del comportamiento humano.

El conocimiento de todos esos factores y su influencia en un colectivo y situación determinada; permitirá realzar un diagnóstico sobre la base de estos conocimientos para generar un modelo que sirva en determinadas situaciones de intervención.

El modelo permitirá conocer la influencia de una serie de factores en la aparición del error, con lo cual se podrá establecer jerarquías o prioridades de atención ente factores, de tal manera que se realicen actividades acertadas y efectivas, direccionado el esfuerzo y los recursos en aquellos que sean significativos, permitiendo incrementar la confiabilidad humana, basada en una realidad actual; así como crear nuevos comportamientos que impulsan la productividad y competitividad de la empresa.

Por último, se cita un concepto del conocido psicólogo Británico James Reason: "Es sumamente crucial que el personal y particularmente sus directivos lleguen a estar más consientes del potencial humano para cometer errores, así como las actividades, del lugar de trabajo y de los factores organizacionales que le dan forma a sus posibilidades y sus consecuencias. Entendiendo cómo y porqué los actos inseguros ocurren, se da el primer paso esencial para el manejo efectivo de errores".

\section{REFERENCIA}

Alvis, A., Pérez, L. y Arrazola G., Elaboración de Panes con Agregado de Harina de Arroz Integral y Modelación de sus Atributos Sensoriales a través de la Metodología de Superficie de Respuesta, Información Tecnológica, 22(5), 29 - 38, (2011). 
Amendola, L. J., Modelo de confiabilidad humana en la gestión de activos. Asociación Española de mantenimiento. Universidad Politécnica de Valencia, España (2002).

Bedford, T. J., Cooke, R., Probalistic risk analysis: foundations and methods. Cambridge University, UK, 408 (2001).

Bermúdez, J., Pérez-García, A. M., Sanjuán, P., Psicología de la personalidad: Teoría e Investigación, Madrid: UNED, Vol. 1. 343-387 (2003).

Cárdenas, S., Fallo humano: la quiebra de un paradigma, Apuntes de psicología, 27 (1), 21 - 51, España (2009).

Bubb, H., Human Reliability: A Key to improved Quality in Manufacturing, Human Factors and Ergonomics in Manufacturing, 15(4), 353-368, (2005).

Cárdenas, S., Fallo humano: la quiebra de un paradigma, Apuntes de psicología, 27 (1), 21 - 51, España (2009).

Čepin, M., Comparasion of Methods for Dependency Determination between Human Failure Events within Human Reliability Analysis, Science and Technology of Nuclear Installations, doi:10.1155/2008/987165, 1-7, (2008).

Collins, R. y Leathley, B., Psychological predispositions to errors in safety, reliability and failure analisys, Safety and Reliability, 14 (3), 6-42 (1995).

Collins, K., Rooney B.L., Smalley, K.J., Havens, S., Funtional Fitness, disease and independence in community-dwelling older adults in western Wisconsin. WMJ, 123(16), 606-10 (2004).

Di Sopra, L., Hypothesis for the construction of vulnerability tables, Third Congress of Emergency and Disaster Medicine, Roma (1983).

Endsley, M.R., Theoretical underpinnings of situation awareness: a critical review. En M.R. Endsley y D.J. Garland, (Eds). Situation Awareness Analysis and Measurement. Mahwah, NJ.LEA (2000).

Hollnagel, E., Human reliability analysis: context and control, Academic Press (1993).

Marseguerra, M., Zio, E. y Librizzi, M., Human Reliability Analysis by Fuzzy “CREAM”, Risk Analysis, 27(1), $137-154,(2007)$.

McCrae, R.R., Costa, P.T. Jr., A five-factor theory of personality. En L.A. Pervin y O.P. John (Eds), Handbook of personality. Theory and reseach (2 Ed). New York: Guildford, 139-153 (1999).

Mulder, G., The heart mental effort. Groningen (discourse). Citado en Drenth, P.J. (1983).

Ramsey, J., Ergonomic factors in task analisys for costumer product safety. Journal of Occupational Accidents, 7. 113-123 (1985).

Rasmussen, J., Human error and the problem of causality in analysis of accidents, Phil. Trns. Royal Society of London, 327, 449-62 (1990).

Sebastián, M.L., Fallo Human: evitando el error. Líneas del tren, 278, 22-23 (2002).

Timiras P.S., Bases fisiológicas del envejecimiento y geriatría. Barcelona: Masson (1997).

Wickens, C.D., Engineering psycology and human performans, Nueva York: Harper Collins (1992).

Wisner, A. (1987). Por dentro do trabalho. Sao Paulo: Editora FTD/Oboré.

Yamamura, T., Yata, K., Yasushi, T., Yamaguchi, H., A basic study on human error in communication network operation. Global Telecomunications Conference and exhibition. Communications Technology for the 1990s beyond. IEEE, 2, 795 - 800 (1989).

Zio, E., Baraldi, P., Librizzi, M., Podofillini, L.fuzzy y Dang, V.N., A fuzzy set-based approach for modelling dependence among human errors, Fuzzy Sets and Systems, ScienceDirect, 160, 1947 - 1964, (2009). 
